CUBO A Mathematical Journal

Vol.12, $N^{\underline{O}} 01$, (15-21). March 2010

\title{
On Some Problems of James Miller
}

\author{
B. Bhowmik, S. Ponnusamy \\ Department of Mathematics, Indian Institute of Technology Madras, \\ Chennai-600 036, India \\ emails: ditya@iitm.ac.in, \\ samy@itm.ac.in \\ AND \\ K.-J. WiRTHS \\ Institut für Analysis, \\ TU Braunschweig, 38106 Braunschweig, Germany \\ email: kjwirths@tu-bs.de
}

\begin{abstract}
We consider the class of meromorphic univalent functions having a simple pole at $p \in(0,1)$ and that map the unit disc onto the exterior of a domain which is starlike with respect to a point $w_{0} \neq 0, \infty$. We denote this class of functions by $\Sigma^{*}\left(p, w_{0}\right)$. In this paper, we find the exact region of variability for the second Taylor coefficient for functions in $\Sigma^{*}\left(p, w_{0}\right)$. In view of this result we rectify some results of James Miller.
\end{abstract}

\section{RESUMEN}

Consideramos la clase de funciones univalentes meromoforficos teniendo un polo simple en $p \in(0,1)$ y la aplicación del disco unitario sobre el exterior de un dominio el cual es estrellado con respecto al punto $w_{0} \neq 0, \infty$. Denotamos esta clase de funciones por $\Sigma^{*}\left(p, w_{0}\right)$. En este artículo encontramos la región exacta de variabilidad del segundo coeficiente de Taylor para funciones in $\Sigma^{*}\left(p, w_{0}\right)$. En vista de estos resultados nosotros rectificamos algunos resultados de James Miller. 
Key words and phrases: Starlike, meromorphic, and Schwarz' functions, Taylor coefficient.

Math. Subj. Class.: 30C45.

\section{Introduction}

Let $\mathbb{D}:=\{z:|z|<1\}$ be the unit disc in the complex plane $\mathbb{C}$. Let $\Sigma^{*}$ denote the class of functions

$$
g(z)=\frac{1}{z}+d_{0}+d_{1} z+d_{2} z^{2}+\cdots
$$

which are univalent and analytic in $\mathbb{D}$ except for the simple pole at $z=0$ and map $\mathbb{D}$ onto a domain whose complement is starlike with respect to the origin. Functions in this class is referred to as the meromorphic starlike functions in $\mathbb{D}$. This class has been studied by Clunie [4] and later an extended version by Pommerenke [10], and many others. Another related class of our interest is the class $S(p)$ of univalent meromorphic functions $f$ in $\mathbb{D}$ with a simple pole at $z=p, p \in(0,1)$, and with the normalization $f(z)=z+\sum_{n=2}^{\infty} a_{n}(f) z^{n}$ for $|z|<p$. If $f \in S(p)$ maps $\mathbb{D}$ onto a domain whose complement with respect to $\overline{\mathbb{C}}$ is convex, then we call $f$ a concave function with pole at $p$ and the class of these functions is denoted by $C o(p)$. In a recent paper, Avkhadiev and Wirths [2] established the region of variability for $a_{n}(f), n \geq 2, f \in C o(p)$ and as a consequence two conjectures of Livingston [7] in 1994 and Avkhadiev, Pommerenke and Wirths [1] were settled.

In this paper, we consider the class $\Sigma^{*}\left(p, w_{0}\right)$ of meromorphically starlike functions $f$ such that $\overline{\mathbb{C}} \backslash f(\mathbb{D})$ is a starlike set with respect to a finite point $w_{0} \neq 0$ and have the standard normalization $f(0)=0=f^{\prime}(0)-1$. We now recall the following analytic characterization for functions in $\Sigma^{*}\left(p, w_{0}\right)$.

Theorem A. $f \in \Sigma^{*}\left(p, w_{0}\right)$ if and only if there is a probability measure $\mu(\zeta)$ on $\partial \mathbb{D}=\{\zeta:|\zeta|=1\}$ so that

$$
f(z)=w_{0}+\frac{p w_{0}}{(z-p)(1-z p)} \exp \left(\int_{\partial \mathbb{D}} 2 \log (1-\zeta z) d \mu(\zeta)\right)
$$

where

$$
w_{0}=-\frac{1}{p+1 / p-2 \int_{|\zeta|=1} \zeta d \mu(\zeta)}
$$

The necessary part of Theorem A has been proved by Miller [9] while the sufficiency part has been established by Yuh Lin [6, Theorem 1]. In [8, 9], Miller discussed a numbers of properties of the class $\Sigma^{*}\left(p, w_{0}\right)$. See also $[3,6,11]$ for some other basic results such as bounds for $\left|f(z)-w_{0}\right|$.

We may state an equivalent formulation of Theorem A (see also [11]). A function $f$ is said to be in $\Sigma^{*}\left(p, w_{0}\right)$ if and only if there exists an analytic function $P(z)$ in $\mathbb{D}$ with $P(0)=1$ and

$$
\operatorname{Re} P(z)>0, z \in \mathbb{D}
$$


where

$$
P(z)=\frac{-z f^{\prime}(z)}{f(z)-w_{0}}-\frac{p}{z-p}+\frac{p z}{1-p z} .
$$

We may write $P(z)$ in the following power series form

$$
P(z)=1+b_{1} z+b_{2} z^{2}+\cdots .
$$

Also, each $f \in \Sigma^{*}\left(p, w_{0}\right)$ has the Taylor expansion

$$
f(z)=z+\sum_{n=2}^{\infty} a_{n}(f) z^{n}, \quad|z|<p .
$$

To recall the next result, we need to introduce a notation. Let $\mathcal{P}\left(b_{1}\right)$ denote the class of analytic functions $P(z)$ satisfying $P(0)=1, P^{\prime}(0)=b_{1}$ and $\operatorname{Re} P(z)>0$ in $\mathbb{D}$.

In 1972, Miller [8] obtained estimations for the second Taylor coefficient $a_{2}(f)$. Indeed, he showed that

Theorem B. If $f(z) \in \Sigma^{*}\left(p, w_{0}\right)$, then the second coefficient is given by

$$
a_{2}(f)=\frac{1}{2} w_{0}\left(b_{2}-p^{2}-\frac{1}{p^{2}}-\frac{1}{w_{0}^{2}}\right)
$$

where $b_{2}$ is the second coefficient of a function in $\mathcal{P}\left(b_{1}\right)$, i.e. the region of variability for $a_{2}(f)$ is contained in the disc

$$
\left|a_{2}(f)+\frac{1}{2} w_{0}\left(p^{2}+\frac{1}{p^{2}}+\frac{1}{w_{0}^{2}}\right)\right| \leq\left|w_{0}\right| .
$$

Further there is a $p_{0}, 0.39<p_{0}<0.61$, such that if $p<p_{0}$, then $\operatorname{Re} a_{2}(f)>0$.

In 1980, Miller [9, Equation (9)] also proved a sharp estimate regarding the second Taylor coefficient. In fact, he showed that

$$
\left|a_{2}(f)-\frac{1+p^{2}}{p}-w_{0}\right| \leq\left|w_{0}\right|, \quad f \in \Sigma^{*}\left(p, w_{0}\right)
$$

The aim of this paper is to find the region of variability for the second coefficient $a_{2}(f)$ of functions in $\Sigma^{*}\left(p, w_{0}\right)$ for any fixed pair $\left(p, w_{0}\right)$. Also we find the exact region of variability for $a_{2}(f)$ for fixed $p$, and as a consequence of this we show that $\operatorname{Re} a_{2}(f)>0$ for all values of $p \in(0,1)$

which Miller did not seem to expect as we see in the last part of Theorem B.

\section{Region of Variability of Second Taylor Coefficients for Functions in $\Sigma^{*}\left(p, w_{0}\right)$}

Theorem 2.1. Let $f \in \Sigma^{*}\left(p, w_{0}\right)$ having the expansion (1.3). Then for a fixed pair $\left(p, w_{0}\right)$, the exact region of variability of the second Taylor coefficient $a_{2}(f)$ is the disc determined by the 
inequality

$$
\begin{aligned}
\left|a_{2}(f)-\left(p+\frac{1}{p}+w_{0}\right)+\frac{1}{4} w_{0}\left(p+\frac{1}{p}+\frac{1}{w_{0}}\right)^{2}\right| & \\
& \leq\left|w_{0}\right|\left(1-\frac{1}{4}\left|p+\frac{1}{p}+\frac{1}{w_{0}}\right|^{2}\right) .
\end{aligned}
$$

Proof. The proof uses the representation formula (1.1), i.e. $f \in \Sigma^{*}\left(p, w_{0}\right)$ if and only if $\operatorname{Re} P(z)>0$ in $\mathbb{D}$ with $P(0)=1$, where $P$ is given by $(1.2)$. Since it is convenient to work with the class of Schwarz functions, we can write each such $P$ as

$$
P(z)=\frac{1+\omega(z)}{1-\omega(z)}, \quad z \in \mathbb{D},
$$

where $\omega: \mathbb{D} \rightarrow \mathbb{D}$ is holomorphic with $\omega(0)=0$ so that $\omega(z)$ has the form

$$
\omega(z)=c_{1} z+c_{2} z^{2}+\cdots
$$

Using (1.2) and the power series representations of $P(z)$ and $f(z)$, it is easy to compute

$$
\left\{\begin{array}{l}
b_{1}=p+\frac{1}{p}+\frac{1}{w_{0}}, \text { and } \\
b_{2}=p^{2}+\frac{1}{p^{2}}+\frac{1}{w_{0}^{2}}+\frac{2 a_{2}(f)}{w_{0}} .
\end{array}\right.
$$

Now eliminating $w_{0}$ from $(2.5)$, we get

$$
b_{2}=p^{2}+\frac{1}{p^{2}}+\left[b_{1}-\left(p+\frac{1}{p}\right)\right]^{2}+2 a_{2}(f)\left[b_{1}-\left(p+\frac{1}{p}\right)\right] .
$$

Using the power series representations of $P(z)$ and $\omega(z)$, it follows by comparing the coefficients of $z$ and $z^{2}$ on both sides that

$$
b_{1}=2 c_{1} \text { and } b_{2}=2\left(c_{1}^{2}+c_{2}\right)
$$

Inserting the above two relations in (2.6), we get

$$
2\left(c_{1}^{2}+c_{2}\right)=p^{2}+\frac{1}{p^{2}}+\left[2 c_{1}-\left(p+\frac{1}{p}\right)\right]^{2}+2 a_{2}(f)\left[2 c_{1}-\left(p+\frac{1}{p}\right)\right] .
$$

Now solving the above equation for $a_{2}(f)$, we get

$$
a_{2}(f)=\frac{1}{p}+p\left(\frac{c_{1}^{2}-c_{2}+p^{2}-2 c_{1} p}{1+p^{2}-2 c_{1} p}\right) .
$$

Now, since $w_{0}$ and $p$ are fixed, we have $c_{1}$ fixed. Hence using the well known estimate $\left|c_{2}\right| \leq 1-\left|c_{1}\right|^{2}$ for unimodular bounded function $\omega(z)$, the last equation results the following estimate

$$
\left|a_{2}(f)-\frac{1}{p}-p\left(\frac{c_{1}^{2}+p^{2}-2 c_{1} p}{1+p^{2}-2 c_{1} p}\right)\right| \leq \frac{p\left(1-\left|c_{1}\right|^{2}\right)}{\left|1+p^{2}-2 c_{1} p\right|} .
$$


Now, as $b_{1}=2 c_{1}$, substituting $c_{1}=\frac{1}{2}\left(p+1 / p+1 / w_{0}\right)$ in the above equation we get the required estimate as given in (2.2). A point on the boundary of the disc described by (2.2) is attained for the unique extremal functions given by (1.2) and (2.3), where

$$
\omega(z)=\frac{z\left(c_{1}+c z\right)}{1+\overline{c_{1}} c z}, \quad|c|=1 .
$$

The points in the interior of the disc described in (2.2) are attained for the same functions, but with $|c|<1$.

Remark. Comparison of Theorem B and Theorem 2.8 below, shows that the exact region of variability of $a_{2}(f)$ found by Miller is for the case $c_{1}=0$ only. A little computation reveals that both variability regions are the same for $c_{1}=0$, i.e.,

$$
\left|a_{2}(f)-\frac{1+p^{2}+p^{4}}{p\left(1+p^{2}\right)}\right| \leq \frac{p}{1+p^{2}} .
$$

This also shows that (1.5) gives the precise region of variability only for the case $c_{1}=0$. In all other cases, the boundaries of the discs described by (1.4) and (1.5) have only one point in common with the disc described by $(2.2)$ because, in the both cases, on the boundaries of the discs described by (1.4) and (1.5), we need $\left|b_{2}\right|=2$. Now, as $b_{2}=2\left(c_{2}+c_{1}^{2}\right)$, this means that $\left|c_{2}+c_{1}{ }^{2}\right|=1$. According to the coefficients bounds for unimodular bounded function, this is only possible for a unique $c_{2}$ if $c_{1} \neq 0$.

In the following theorem, we describe the exact region of variability of the second Taylor coefficient of $f \in \Sigma^{*}\left(p, w_{0}\right)$, where only $p$ is fixed.

Theorem 2.8. Let $f \in \Sigma^{*}\left(p, w_{0}\right)$ having the expansion (1.3) and let $p$ be fixed. Then the exact set of variability of the second Taylor coefficient $a_{2}(f)$ is given by

$$
\left|a_{2}(f)-1 / p\right| \leq p .
$$

Proof. We may rewrite (2.7) as

$$
a_{2}(f)=\frac{1}{p}+p M
$$

where

$$
M=\frac{c_{1}^{2}-c_{2}+p^{2}-2 c_{1} p}{1+p^{2}-2 c_{1} p} .
$$

We wish to prove that $|M| \leq 1$. Since $\omega^{\prime}(0)=c_{1}$, we have $\left|c_{1}\right| \leq 1$.

Now we fix $c_{1} \in \overline{\mathbb{D}}$. Then $c_{1}^{2}-c_{2}$ varies in the closed disc

$$
\Delta\left(c_{1}\right):=\left\{z:\left|z-c_{1}^{2}\right| \leq 1-\left|c_{1}\right|^{2}\right\} .
$$


The map

$$
T(\zeta)=\frac{\zeta+p^{2}-2 c_{1} p}{1+p^{2}-2 c_{1} p}
$$

maps the disc $\Delta\left(c_{1}\right)$ onto the disc with center

$$
\frac{c_{1}^{2}+p^{2}-2 c_{1} p}{1+p^{2}-2 c_{1} p}
$$

and radius

$$
\frac{1-\left|c_{1}\right|^{2}}{\left|1+p^{2}-2 c_{1} p\right|} .
$$

Therefore, in order to prove $|M| \leq 1$, it suffices to show that

$$
\left|\frac{c_{1}^{2}+p^{2}-2 c_{1} p}{1+p^{2}-2 c_{1} p}\right|+\frac{1-\left|c_{1}\right|^{2}}{\left|1+p^{2}-2 c_{1} p\right|} \leq 1 .
$$

This is equivalent to

$$
\left|c_{1}-p\right|^{2}+1-\left|c_{1}\right|^{2}=\operatorname{Re}\left(1+p^{2}-2 c_{1} p\right) \leq\left|1+p^{2}-2 c_{1} p\right| .
$$

We see that equality is attained in the above inequality if and only if $c_{1}$ is real. Now for real $c_{1}$, we have

$$
T\left(\Delta\left(c_{1}\right)\right)=\overline{\mathbb{D}} \text { if and only if } c_{1}=p \text { or } w_{0}=\frac{-p}{1-p^{2}} .
$$

Hence the extremal functions for the inequality (2.9) are given by (1.2) with $P(z)$ as in $(2.3)$ with

$$
\omega(z)=\frac{z(p+c z)}{1+p c z},|c|=1
$$

and the points in the interior of the disc described by (2.9) are attained for the same functions, but with $|c|<1$. We observe that for real $c_{1}$ we can obtain $M=1$ only for $c_{2}=c_{1}^{2}-1$. This results in other starlike centers, but the extremal function is always the same, since $a_{2}(f)=p+1 / p$ is attained in the class $S(p)$ only for $f(z)=z /((1-z p)(1-z / p))$, see for instance [5].

Remark. This result ensures us that $\operatorname{Re} a_{2}(f)>0$ for all $p \in(0,1)$. In the article [8, Theorem 1], Miller hoped for a possibility that for $p>.61$, the real part of $a_{2}(f)$ may be negative. But in view of our theorem we conclude that his hope was in vain.

Remark. In [9], Miller has obtained an estimate for the real part of the third coefficient $a_{3}(f)$ for all $p$. However, in geometric function theory, the classical question of finding the exact region of variability for $a_{n}(f), n \geq 3, f \in \Sigma^{*}\left(p, w_{0}\right)$, remains an open problem.

Received: March, 2008. Revised: September, 2009. 


\section{References}

[1] Avkhadiev, F.G., Pommerenke, C. And Wirths, K.-J., On the coefficients of concave univalent functions, Math. Nachr., 271(2004), 3-9.

[2] Avkhadiev, F.G. And Wirths, K.-J., A proof of the Livingston conjecture, Forum math., 19(2007), 149-158.

[3] Bhowmik, B. And Ponnusamy, S., Coefficient inequalities for concave and meromorphically starlike univalent functions, Ann. Polon. Math., 93(2008), 177-186.

[4] Clunie, J., On meromorphic schlicht functions, J. London Math. Soc., 34(1959), 215-216.

[5] Jenkins, J.A., On a conjecture of Goodman concerning meromorphic univalent functions, Michigan Math. J., 9(1962), 25-27.

[6] Yun Lin, C., On the representation formulas for the functions in the class $\Sigma^{*}\left(p, w_{0}\right)$, Proc. Amer. Math. Soc., 103(1988), 517-520.

[7] Livingston, A.E., Convex meromorphic mappings, Ann. Polon Math., 59(3)(1994), 275291.

[8] Miller, J., Starlike meromorphic functions, Proc. Amer. Math. Soc., 31(1972), 446-452.

[9] Miller, J., Convex and starlike meromorphic functions, Proc. Amer. Math. Soc., 80(1980), $607-613$.

[10] Pommerenke, C., On meromorphic starlike functions, Pacific J. Math., 13(1963), 221-235.

[11] Yulin, Z. AND Owa, S., Some remarks on a class of meromorphic starlike functions, Indian J. pure appl. Math., 21(9)(1990), 833-840. 